\title{
Limited Clinical Utility of Chest Radiography in Asymptomatic Patients after Interventional Radiology- Performed Ultrasound-Guided Thoracentesis: Analysis of 3,022 Consecutive Patients
}

\author{
Jacob J. Bundy ${ }^{1}$ Anthony N. Hage ${ }^{2}$ Ruple Jairath ${ }^{3}$ Albert Jiao ${ }^{4}$ Vibhor Wadhwa ${ }^{5}$ \\ Narendra B. Gutta ${ }^{6}$ Ravi N. Srinivasa ${ }^{7} \quad$ Joseph J. Gemmete ${ }^{1} \quad$ Eric J. Monroe ${ }^{8}$ \\ Jeffrey Forris Beecham Chick $^{9}$
}

\footnotetext{
${ }^{1}$ Division of Vascular and Interventional Radiology, Department of Radiology, University of Michigan Health System, Ann Arbor, Michigan, United States

2Department of Radiology, Thomas Jefferson University Hospitals, Philadelphia, Pennsylvania, United States

${ }^{3}$ Department of Neuroscience, University of Evansville, Evansville, Indiana, United States

${ }^{4}$ Michigan State University College of Human Medicine, Grand Rapids, Michigan, United States

${ }^{5}$ Division of Interventional Radiology, NewYork-Presbyterian/Weill Cornell Medical Center, New York, NY 10065, United States

${ }^{6}$ Department of Radiology, University of Arkansas for Medical Sciences, Little Rock, Arkansas, United States

${ }^{7}$ Department of Interventional Radiology, University of California Los Angeles, Los Angeles, California, United States

8 Section of Interventional Radiology, Department of Radiology, University of Washington, Seattle Children's Hospital, Seattle, Washington, United States

${ }^{9}$ Division of Interventional Radiology, Department of Radiology, University of Washington, 1959 Northeast Pacific Street, Seattle, Washington, United States
}

\begin{abstract}
Address for correspondence Jeffrey Forris Beecham Chick, MD, MPH, Division of Interventional Radiology, Department of Radiology, University of Washington, 1959 Northeast Pacific Street, Seattle, WA 98195, United States (e-mail: jeffreychick@gmail.com).
\end{abstract}

J Clin Interv Radiol ISVIR 2021;5:86-90.

\begin{abstract}
Keywords

- ultrasound

- thoracentesis

- pneumothorax

- chest radiograph

- chest tube placement

- drainage catheter placement

- interventional radiology

Purpose The aim of this study was to report the utility of chest radiography following interventional radiology-performed ultrasound-guided thoracentesis.

Materials and Methods A total of 3,998 patients underwent thoracentesis between 2003 and 2018 at two institutions. A total of 3,022 (75.6\%) patients were older than 18 years old, underwent interventional radiology-performed ultrasound-guided thoracentesis, and had same-day post-procedure chest radiograph evaluation. Patient age (years), laterality of thoracentesis, procedural technical success, volume of fluid removed $(\mathrm{mL})$, method of post-procedure chest imaging, absence or presence of pneumothorax, pneumothorax size $(\mathrm{mm})$, pneumothorax management measures, and clinical outcomes were recorded. Technical success was defined as successful aspiration of pleural fluid. Post-procedure clinical outcomes included new patient-perceived dyspnea and hypoxia (oxygen saturations $<90 \%$ on room air). Costs associated with radiographs were estimated using Medicare and Medicaid fee schedules.
\end{abstract}

DOI https://doi.org/ 10.1055/s-0041-1723096 ISSN 2457-0214.
(C) 2021. Indian Society of Vascular and Interventional Radiology. This is an open access article published by Thieme under the terms of the Creative Commons Attribution-NonDerivative-NonCommercial-License, permitting copying and reproduction so long as the original work is given appropriate credit. Contents may not be used for commercial purposes, or adapted, remixed, transformed or built upon. (https://creativecommons.org/licenses/by-nc-nd/4.0/).

Thieme Medical and Scientific Publishers Pvt. Ltd. A-12, 2nd Floor, Sector 2, Noida-201301 UP, India 
Results Mean age was $56.7 \pm 15.5$ years. Interventional radiology-performed ultrasound-guided thoracentesis was performed on the left ( $n=1,531 ; 50.7 \%)$, right $(n=$ 1,$477 ; 48.9 \%$ ), and bilaterally ( $n=14 ; 0.5 \%$ ) using 5 -French catheters. Technical success was $100 \%(n=3,022)$. Mean volume of $940 \pm 550 \mathrm{~mL}$ of fluid was removed. Postprocedure imaging was performed in the form of posteroanterior (PA) $(2.6 \% ; 78 / 3,022)$, anteroposterior (AP) $(17.0 \%$; 513/3,022), PA and lateral $(77.9 \% ; 2,355 / 3,022)$, or PA, lateral, and left lateral decubitus $(2.5 \% ; 76 / 3,022)$ chest radiographs. Post-procedural pneumothorax was identified in $21(0.69 \%)$ patients. Mean pneumothorax size, measured on chest radiograph as the longest distance from the chest wall to the lung, was $18.8 \pm 10.2 \mathrm{~mm}$ (range: $5.0-35.0 \mathrm{~mm}$ ). Of the 21 pneumothoraces, 7 (33.3\%) were asymptomatic, resolved spontaneously, and had a mean size of $6.4 \pm 2.4 \mathrm{~mm}$. Fourteen pneumothoraces, of mean size $25.0 \pm 5.8 \mathrm{~mm}$, required management with a pleural drainage catheter (66.6\%). The overall incidence of pneumothorax requiring pleural drainage catheter placement following interventional radiology-performed ultrasound-guided thoracentesis was $0.46 \%(14 / 3,022)$. Of the patients requiring drainage catheter placement, $12 / 14(85.7 \%)$ and 13/14 (92.9\%) had dyspnea and hypoxia, respectively. Potential costs to Medicare and Medicaid, for chest radiographs, in this study, were $\$ 27,547$ and $\$ 10,581$, respectively.

Conclusion The incidence of clinically significant pneumothorax requiring catheter drainage following interventional radiology-operated ultrasound-guided thoracentesis is exceedingly low $(0.46 \%)$, and routine post-procedure chest radiographs in asymptomatic patients provide little value. Reserving post-procedure chest radiographs for patients with post-procedure dyspnea or hypoxia will result in more efficient resource utilization and health care cost savings.

\section{Introduction}

Pleural effusions are commonly treated with thoracentesis as part of diagnostic and therapeutic management strategies. In the United States, a pleural effusion is diagnosed in 1.5 million patients annually. ${ }^{1}$ Thoracentesis involves percutaneous aspiration of pleural fluid through the chest wall. Common adverse events include pneumothorax (4-39\%), cough (24\%), dyspnea (15\%), chest pain (5\%), and vasovagal reactions (3\%), ${ }^{2,3}$ Less common complications include inadvertent liver or splenic laceration, hemorrhage, infection, and re-expansion pulmonary edema., ${ }^{2,3}$

Ultrasound guidance is increasingly utilized for thoracentesis as it has been shown to reduce the risk of pneumothoraces ( $<3 \%$ overall with a $90 \%$ risk reduction compared with nonultrasound-guided procedures) and has been associated with lower total hospital costs. Despite the reduction in pneumothoraces, it is still common practice to obtain routine post-procedure chest radiographs after thoracentesis. There are no consensus or societal guidelines that recommend or refute the utility of chest radiographs after thoracentesis.

The purpose of this study was to report the clinical utility of chest radiography following interventional radiology-performed ultrasound-guided thoracentesis.

\section{Materials and Methods}

\section{Study Design}

This study was conducted with Institutional Review Board approval (HUM00151864 and 18-3202) at two institutions and complied with the Health Insurance Portability and Accountability Act. Informed consent was not required for this retrospective study. This study was assessed using the Strengthening the Reporting of Observational studies in Epidemiology (STROBE) guidelines. ${ }^{4}$ Patients with pleural effusions identified on chest radiography or computed tomography and who underwent thoracentesis between 2003 and 2018 were identified.

\section{Inclusion and Exclusion Criteria}

A total of 3,998 patients underwent thoracentesis. A total of 3,022 (75.6\%) patients were older than 18 years old, underwent interventional radiology-performed ultrasound-guided thoracentesis, and had same-day ( $<24$ hours) post-procedure chest radiograph evaluation. Patients younger than 18 years old, those who underwent thoracentesis by noninterventional radiology specialties, or those without same-day chest radiograph evaluation were excluded $(n=976)$. The decision to obtain a radiograph was based on the individual provider and patient's clinical status. 
Limited Clinical Utility of Chest Radiography in Asymptomatic Patients after Interventional Radiology-Performed

\section{Thoracentesis and Pleural Drainage Catheter Placement Techniques}

Thoracentesis procedures have been previously described., ${ }^{5,6} \mathrm{All}$ procedures were performed by an interventional radiologist using ultrasound guidance. Ultrasound-guided thoracentesis was performed using standard operating procedure and a generic 5-French catheter (end-hole, nonvalved) with syringe or vacuum container at the operator's discretion. Procedures were terminated when $1.5 \mathrm{~L}$ of fluid was removed; patient experienced discomfort or excessive coughing, or at operator discretion. Pleural drainage catheters were placed to treat the pneumothorax, under fluoroscopic or computed tomography-guidance, based on operator's discretion. Catheter size was based on operator's discretion.

\section{Variables and Definitions}

Patient age (years), laterality of thoracentesis (left, right, or bilateral), procedural technical success, volume of fluid removed $(\mathrm{mL})$, method of post-procedure chest imaging (anteroposterior [AP or portable], posteroanterior [PA or one view], PA and lateral [two view], or PA, lateral, and left lateral decubitus [three view] chest radiography), absence or presence of pneumothorax, pneumothorax size ( $\mathrm{mm})$, pneumothorax management measures, and post-procedure clinical outcomes were recorded. Technical success was defined as successful aspiration of pleural fluid. Method of post-procedure chest imaging was determined by operator preference. Pneumothorax was defined as any abnormal air in the pleural cavity. Pneumothorax size was recorded based on the longest distance from the chest wall to the lung. ${ }^{7,8}$ Pneumothorax management measures included repeat chest imaging or placement of a pleural drainage catheter. The pleural drainage catheter size was recorded in French. Post-procedure clinical outcomes included new patient-perceived dyspnea and hypoxia (oxygen saturations $<90 \%$ on room air).

\section{Cost Estimation}

Current Procedural Terminology (CPT) codes for one-, two-, and three-view chest radiographs were obtained from publicly available government reimbursement schedules. CPT codes included 71,045 (chest one view), 71,046 (chest two views), and 71,047 (chest three views). Mean costs associated with chest radiographs after thoracentesis were estimated using Medicare and Medicaid fee schedules. ${ }^{9,10}$ Medicare reimbursement of one-, two-, and three-view radiographs were $\$ 23.78, \$ 30.19$, and $\$ 37.95$, respectively, with weighted mean of $\$ 29.84 .{ }^{9}$ Medicaid reimbursements for these three examinations were $\$ 13.87$, \$17.63, and $\$ 22.19$, with weighted mean of $\$ 17.42 .^{10}$

\section{Statistical Analyses}

All statistical analyses, including means, percentages, and standard deviations, were calculated using $\mathrm{R}$ software version 3.2.2 (R Core Team).

\section{Results}

A total of 3,022 (75.6\%) patients were older than 18 years old, underwent interventional radiology-performed ultrasound-guided thoracentesis, and had same-day post-procedure chest radiograph evaluation. Mean patient age was $56.7 \pm 15.5$ years. Laterality of thoracentesis, volume of fluid removed, method of post-procedure chest imaging, absence or presence of pneumothorax, and pneumothorax size are shown in - Table 1 . Of the total 3,022 procedures, 1,531 (50.7\%; 1,531/3,022), 1,477 (48.9\%; 1,477/3,022), and $14(0.5 \%$; $14 / 3,022$ ) were performed on left, right, or bilaterally in a single session. Technical success was $100 \%(n=3,022)$. A mean volume of $940 \pm 550 \mathrm{~mL}$ fluid was removed in a single session. Post-procedure imaging was performed in the form of PA (2.6\%; 78/3,022), AP (17.0\%; 513/3,022), PA and lateral (77.9\%; $2,355 / 3,022)$, or PA, lateral, and left lateral decubitus $(2.5 \%$; $76 / 3,022$ ) chest radiographs. Post-procedural pneumothorax was identified in 21 patients with a total incidence of $0.69 \%$ ( $n=21 / 3,022$ ). Mean, pneumothorax size, measured on chest radiograph as the longest distance from the chest wall to the pleural reflection, was $18.8 \pm 10.2 \mathrm{~mm}$ (range: $5.0-35.0 \mathrm{~mm}$ ).

Pneumothorax management and clinical outcomes are shown in - Table 2 . Of the 21 pneumothoraces, seven (33.3\%) were asymptomatic and resolved spontaneously and had a mean size of $6.4 \pm 2.4 \mathrm{~mm}$. Fourteen pneumothoraces, of

Table 1 Thoracenteses and post-procedural chest radiograph results

\begin{tabular}{|l|l|l|}
\hline Variable & Outcome \\
\hline \multirow{2}{*}{ Laterality of effusion } & Left & $1,531(50.7 \%)$ \\
\cline { 2 - 3 } & Right & $1,477(48.9 \%)$ \\
\cline { 2 - 3 } & Bilateral & $14(0.5 \%)$ \\
\hline Mean volume removed & $940 \mathrm{~mL}(550 \mathrm{~mL})$ \\
\hline Chest radiograph technique & Portable & $78(2.6 \%)$ \\
\cline { 2 - 3 } & One view & $513(17.0 \%)$ \\
\cline { 2 - 3 } & Two views & $2,355(77.9 \%)$ \\
\cline { 2 - 3 } & $\begin{array}{l}\text { Three } \\
\text { views }\end{array}$ & $76(2.5 \%)$ \\
\hline Post-procedural pneumothorax & $21(0.69 \%)$ \\
\hline Mean pneumothorax size & $18.8(10.2 \mathrm{~mm})$ \\
\hline
\end{tabular}

Numerical measures are summarized as mean (standard deviation) and categorical variables are shown as number of cases (percentage of 3022).

Table 2 Outcomes of pneumothoraces

\begin{tabular}{|l|l|l|}
\hline \multicolumn{2}{|l|}{ Variable } & Outcome \\
\hline $\begin{array}{l}\text { Method of } \\
\text { pneumothorax } \\
\text { resolution }\end{array}$ & Spontaneous & $7(33.3 \%)$ \\
\cline { 2 - 3 } & $\begin{array}{l}\text { 10-French } \\
\text { pigtail catheter }\end{array}$ & $9(42.8 \%)$ \\
\cline { 2 - 3 } & Chest tube & $5(23.8 \%)$ \\
\hline \multirow{3}{*}{$\begin{array}{l}\text { Chest tube size } \\
\text { Mean pneumotho- }\end{array}$} & $\begin{array}{l}\text { Not requiring } \\
\text { intervention }\end{array}$ & $2(9.5 \%)$ \\
\cline { 2 - 3 } & 21-French & $2(9.5 \%)$ \\
\cline { 2 - 3 } & $\begin{array}{l}\text { 22-French } \\
\text { Requiring } \\
\text { intervention }\end{array}$ & $25.0 \mathrm{~mm}(5.8 \mathrm{~mm})$ \\
\hline
\end{tabular}

Numerical measures are summarized as mean (standard deviation) and categorical variables are shown as number of cases (percentage of 21). 
mean size $25.0 \pm 5.8 \mathrm{~mm}$, required management with a pleural drainage catheter (66.6\%; 14/21). Nine (42.8\%; 9/21) were managed with 10 -French pigtail placement and five $(23.8 \%$; $5 / 21$ ) were managed with larger chest tube placement, based on operator discretion. Two chest tubes were 20-French, two were 21-French, and one was 22-French. The overall incidence of pneumothorax requiring pleural drainage catheter placement following interventional radiology-performed ultrasound-guided thoracentesis was $0.46 \%(14 / 3,022)$. Of the patients requiring drainage catheter placement, 12 (85.7\%) and 13 (92.9) had dyspnea and hypoxia, respectively.

There were 937 patients older than 65 years; thus, the cost to the Medicare population was $\$ 27,547$. The proportion of adults in Medicaid was $20.1 \%$ at the time of this manuscript, equating to 607 patients in this study; thus, the cost to Medicaid was \$10,581.

\section{Discussion}

With the advent of ultrasound-guided thoracentesis, the overall incidence and risk of post-procedural complications have been significantly reduced, especially pneumothorax rates. ${ }^{11}$ In this study, the overall incidence of iatrogenic pneumothorax following interventional radiology-performed ultrasound-guided thoracentesis was $0.69 \%$. Previous studies have reported the incidence of thoracentesis-related pneumothorax ranging from 4 to $39 \%$ without the use of ultrasound ${ }^{2,3}$ and more recent, smaller investigations have found the pneumothorax rates ranging from 0 to $3 \%$ with the use of ultrasound..$^{5,6,12,13}$ Overall, ultrasound guidance has led to a $90 \%$ reduction in the incidence of iatrogenic pneumothorax compared with nonimage-guided thoracentesis.

Recently, two large studies of 9,320 and 3,067 patients reported a 0.61 and $0.62 \%$ incidence of thoracentesis-related pneumothorax, respectively, which is similar to the $0.69 \%$ reported in this study. ${ }^{14,15}$ Ault et al included thoracenteses that were performed, or supervised, by a single internist, whereas this study included thoracenteses performed by interventional radiologists. ${ }^{14}$ Cho et al described thoracenteses performed in the emergency department by emergency medicine physicians, internists, and radiologists. ${ }^{15}$ Taken as a whole, considering the various clinical situations and operators, the similar low rate of pneumothorax suggests the marked efficacy of ultrasound and resultant consistency across operator specialties.

When rare post-procedural pneumothoraces did occur, $33.3 \%$ (7/21) were asymptomatic and resolved spontaneously. About $0.46 \%(14 / 3,022)$ of patients who underwent interventional radiology-performed ultrasound-guided thoracentesis required pleural drainage catheter placement. Moreover, of the patients requiring drainage catheter placement, the majority of patients was symptomatic with $12 / 14$ (85.7\%) and 13/14 (92.9\%) experiencing new dyspnea and hypoxia, respectively. Cho et al reported a similarly low rate of chest tube insertion in $36.8 \%$ of those patients $(0.2 \%$ overall) for the management of iatrogenic pneumothorax in the emergency department. ${ }^{15}$ Other studies reported chest tube insertion following $1.7 \%$ of all thoracentesis procedures to evacuate symptomatic pneumothoraces. ${ }^{6}$ Pneumothorax identification, however, does not lead to patient benefit in all circumstances and may increase costs related to unnecessary observation or treatment. In cases of asymptomatic pneumothorax ex vacuo, or pneumothorax resulting from failed re-expansion of noncompliant lung rather than disruption of the visceral pleura, drain placement provides little benefit and may expose patients to iatrogenic harm. ${ }^{16-18}$

Despite the low incidence of iatrogenic pneumothorax following ultrasound-guided thoracentesis (0.69\%) and even lower requirement rates of pleural drainage catheter placement $(0.46 \%)$, it is still common clinical practice to obtain a routine post-thoracentesis chest radiograph regardless of patient symptoms. Because follow-up chest radiography protocol is not standardized, various combinations of portable, single-, or multiple-view radiographs without or with expiration are performed. In this study, chest radiograph was performed in the form of portable (78; $2.6 \%)$, one view (513; $17.0 \%)$, two views (2,355; 77.9\%), and three views (76; $2.5 \%)$. A single-view chest radiograph costs $\$ 23.78$, while a two-view chest radiograph costs $\$ 30.19$ to Medicare. ${ }^{9,10}$ Thoracentesis procedures are performed in 173,000 patients annually with most patients receiving some form of post-procedural follow-up chest radiograph. ${ }^{19-23}$ If the same proportion of patients in the United States qualified for Medicare and Medicaid as those in this study, there would be 53,640 and 34,600 thoracentesis patients receiving Medicare and Medicaid benefits, respectively. At a minimum, if all patients received a single-view chest radiograph at $\$ 23.78$, the total cost to Medicare would be $\$ 1.28$ million per year. Total cost to Medicaid at $\$ 13.87$ would equate to $\$ 480,000$ per year. While computed tomography remains the gold standard for the diagnosis of pneumothorax, the utilization of point-of-care ultrasound may provide a cost-effective alternative to screen for pneumothorax in symptomatic patients following thoracentesis. ${ }^{24}$

The routine practice of obtaining immediate post-procedure follow-up chest radiograph comes with little tangible patient benefit and at significant health care costs. In asymptomatic patients, without dyspnea or hypoxia, routine chest radiographs are of limited clinical utility immediately after interventional radiology-performed ultrasound-guided thoracentesis.

Limitations of the present study relate in part to the retrospective study design. Mechanically ventilated patients are at an increased risk of pneumothorax following thoracentesis compared with nonventilated patients and, as a result, the complications within the present analysis may have occurred disproportionally among the ventilated population. ${ }^{12}$ More broadly, limited data on the indication for thoracentesis and the etiology of the pleural effusions in each patient inhibits identification of clinical scenarios or patient subpopulations where routine post-procedure radiography may be value-added. Chest radiograph follow-up technique was not standardized with some patients receiving supine portable or single-view radiographs that may have limited detection of pneumothorax. Furthermore, since all thoracentesis procedures were performed by interventional radiologists, with expertise in image-guided interventions, 
the generalizability of the results may be limited among other medical specialties. Further, the level of training and expertise of the interventional radiologists performing the procedures was not controlled for and may have been correlated with procedural complications. While presumed to be significant, practice costs related to routine post-thoracentesis radiography, including potentially unnecessary monitoring and treatment of asymptomatic patients, are beyond the scope of the economic analysis presented.

\section{Conclusion}

The incidence of clinically significant pneumothorax requiring catheter drainage following interventional radiology-operated ultrasound-guided thoracentesis is incredibly low $(0.46 \%)$, and routine post-procedure chest radiographs in asymptomatic patients provide little value. Reserving post-procedure chest radiographs for patients with new post-procedure dyspnea or hypoxia will result in more efficient resource utilization and health care cost savings.

\section{Note}

All procedures performed in studies involving human participants were in accordance with the ethical standards of the institutional and/or national research committee and with the 1964 Helsinki Declaration and its later amendments or comparable ethical standards.

For this type of study, formal consent was not required. This study was Institutional Review Board-approved and the need for informed consent was waived.

For this type of study consent for publication was not required.

\section{Conflict of Interest}

None declared.

\section{References}

1 Light RW. Clinical practice. Pleural effusion. N Engl J Med 2002;346(25):1971-1977

2 Duncan DR, Morgenthaler TI, Ryu JH, Daniels CE. Reducing iatrogenic risk in thoracentesis: establishing best practice via experiential training in a zero-risk environment. Chest 2009;135(5):1315-1320

3 Grogan DR, Irwin RS, Channick R, et al. Complications associated with thoracentesis. A prospective, randomized study comparing three different methods. Arch Intern Med 1990;150(4):873-877

4 von Elm E, Altman DG, Egger M, Pocock SJ, Gøtzsche PC, Vandenbroucke JP; STROBE Initiative. The Strengthening the Reporting of Observational Studies in Epidemiology (STROBE) Statement: guidelines for reporting observational studies. Int J Surg 2014;12(12):1495-1499
5 Barnes TW, Morgenthaler TI, Olson EJ, Hesley GK, Decker PA, Ryu JH. Sonographically guided thoracentesis and rate of pneumothorax. J Clin Ultrasound 2005;33(9):442-446

6 Jones PW, Moyers JP, Rogers JT, Rodriguez RM, Lee YCG, Light RW. Ultrasound-guided thoracentesis: is it a safer method? Chest 2003;123(2):418-423

7 MacDuff A, Arnold A, Harvey J; BTS Pleural Disease Guideline Group. Management of spontaneous pneumothorax: British Thoracic Society pleural disease guideline 2010. Thorax 2010;65, Suppl 2):ii18-ii31

8 Baumann MH, Strange C, Heffner JE, et al; AACP Pneumothorax Consensus Group. Management of spontaneous pneumothorax: an American College of Chest Physicians Delphi consensus statement. Chest 2001;119(2):590-602

9 Physician Fee Schedule Search. Available from: https://www. cms.gov/apps/physician-fee-schedule/search/search-criteria. aspx. Accessed May 26, 2020

10 MDHHS - Physicians/Practitioners/Medical Clinics. Available from: https://www.michigan.gov/mdhhs/0,5885,7-33971551_2945_42542_42543_42546_42551-151022-,00.html. Accessed May 26, 2020

11 Mercaldi CJ, Lanes SF. Ultrasound guidance decreases complications and improves the cost of care among patients undergoing thoracentesis and paracentesis. Chest 2013;143(2):532-538

12 Gordon CE, Feller-Kopman D, Balk EM, Smetana GW. Pneumothorax following thoracentesis: a systematic review and meta-analysis. Arch Intern Med 2010;170(4):332-339

13 Raptopoulos V, Davis LM, Lee G, Umali C, Lew R, Irwin RS. Factors affecting the development of pneumothorax associated with thoracentesis. AJR Am J Roentgenol 1991;156(5):917-920

14 Ault MJ, Rosen BT, Scher J, Feinglass J, Barsuk JH. Thoracentesis outcomes: a 12-year experience. Thorax 2015;70(2):127-132

15 Cho HY, Ko BS, Choi HJ, et al. Incidence and risk factors of iatrogenic pneumothorax after thoracentesis in emergency department settings. J Thorac Dis 2017;9(10):3728-3734

16 Ponrartana S, Laberge JM, Kerlan RK, Wilson MW, Gordon RL. Management of patients with "ex vacuo" pneumothorax after thoracentesis. Acad Radiol 2005;12(8):980-986

17 Chang YC, Patz EF Jr, Goodman PC. Pneumothorax after small-bore catheter placement for malignant pleural effusions. AJR Am J Roentgenol 1996;166(5):1049-1051

18 Boland GW, Gazelle GS, Girard MJ, Mueller PR. Asymptomatic hydropneumothorax after therapeutic thoracentesis for malignant pleural effusions. AJR Am J Roentgenol 1998;170(4):943-946

19 Daniels CE, Ryu JH. Improving the safety of thoracentesis. Curr Opin Pulm Med 2011;17(4):232-236

20 Petersen WG, Zimmerman R. Limited utility of chest radiograph after thoracentesis. Chest 2000;117(4):1038-1042

21 Owings MF, Kozak LJ. Ambulatory and inpatient procedures in the United States, 1996. Vital Health Stat 13 1998;(139):1-119

22 Light RW. Pleural effusions. Med Clin North Am 2011;95(6):1055-1070

23 Feller-Kopman D. Ultrasound-guided thoracentesis. Chest 2006;129(6):1709-1714

24 Husain LF, Hagopian L, Wayman D, Baker WE, Carmody KA. Sonographic diagnosis of pneumothorax. J Emerg Trauma Shock 2012;5(1):76-81 